enthaltenen Seife aufweist, wird filtriert und das erhaltene Filtrat unter Verwendung von Phenolphtalein als Indikator austitriert.

Fahrion's Arbeit enthält schliesslich noch Auseinandersetzungen über einige regelmälsig auftretende Unstimmigkeiten bei Seifenanalysen. So bleibt die Summe der direkt bestimmten Bestandteile meist unter $100 \%$; die Gesamtmenge der Alkalien ist, grösser als die aus Fettsäuregehalt und Verseifungszahl der Fettsäuren berechnete, und dennoch enthält die Seife kein freies Alkali. Wegen dieser Dinge verweise ich auf das Original.

2. Auf Pharmazie bez ìgliche Methoden. Von

H. Mühe.

\title{
Über eine vergleichende Untersuchung verschiedener Kreoline
} des Handels berichtet A. Gaw a low sk $\mathrm{i}^{1}$ ). Zur schnellen Wertbestimmung von Handels-Kreolinen führt der Verfasser folgende Bestimmungen aus: Bestimmung des spezifischen Gewichtes; Reaktion gegen Phenolphtalein; Bestimmung des Wassergehaltes, des Gehaltes an Phenolen und an Harz- und Fettseifen. Zur Bestimmung des Wassers, der Phenole und der Seifen bringt man in eine Messröhre von $30 \mathrm{cc}$ Inhalt, welche in $0,1 c c$ geteilt ist, $10 \mathrm{cc}$ Kreolin, fügt $10 \mathrm{cc}$ Chlorkalziumlösung (bereitet aus $25 \mathrm{~g}$ entwässertem Chlorkalzium und $100 \mathrm{cc}$ Wasser) zu und schüttelt; hierauf bringt man noch $10 c c$ Benzin in die Röhre, schüttelt kräftig durch und stellt gut verschlossen einen halben bis einen Tag bei Seite. Es bilden sich drei Schichten: Zu unterst eine ungefärbte Wasserschicht, darüber eine lehmiggelbe Breischicht and oben eine tief braune Benzinlösung. Die Zunahme der Benzinschicht gibt das Volumen der Phenole, die Zunahme der Wasserschicht das Volumen des Wassers in dem Kreolin an. Einen kleinen Teil der Benzinschicht befreit man durch Erhitzen im Wasserbade vom Benzin, behandelt den Rückstand mit Wasserstoff im statu nascendi und prüft das Gas nach 12 bis 24 Stunden mit Papier, welches mit Bleiessig getränkt ist; bräunt sich das Papier, so liegen sulfonierte Phenole vor. Nach der oben besprochenen Methode findet man den Gehalt an Seifen in den Kreolinen, wenn man die für Wasser und Phenole ermittelten Zahlen von 100 abzieht. Der

1) Zeitschrift d. allgem. üsterr. Apotheker-Vereins 58, 1003. 
Verfasser ${ }^{1}$ ) hat nun aber gefunden, dass manclie Kreoline Alkalifettseife, Alkaliresinate oder Gemische von beiden enthalten. Um die genannten Seifen von einander zu unterscheiden, schüttelt man nach dem Vorschlage des Verfassers $10 \mathrm{cc}$ des zu untersucbenden Kreolins mit $10 \mathrm{cc}$ Benzin und $10 \mathrm{cc}$ Wasser aus. Von der wässerigen Schicht bringt man $5 c c$ in ein Reagensglas, versetzt (nach Schädler) mit konzentrierter Salpetersäure und erhitzt zum Sieden. Bei Gegenwart von Harzen soll ein charakteristischer Harzgeruch auftreten, während sich die Fettsäuren der Alkalifettseifen beim. Erkalten als olige oder starre Fettschicht an der Oberfiäche abscheiden.

Über die Prüfung von Äther und Narkoseäther veröffentlicht W. Wobbe ${ }^{2}$ ) eine eingehende Abhandlung, Der Verfasser hat die Prüfungsvorschriften von Äther und Narkoseäther kritisch nachgeprüft und besprochen; er hat dabei nicht nur die einschlägige Literatur, sondern auch die vier jüngsten europäischen Pharmakopöen benutzt, nämlich die schweizerische, die englische, die deutsche, die schwedische and den Entwurf zur vierten schweizerischen Pharmakopöe. Die Versuche des Verfassers ergaben im allgemeinen, dass eine Anzahl von Abänderungsvorschlägen für die Prüfung von Äther, wie sie in der Literatur veröffentlicht worden sind, keine Berechtigung haben, weiterhin aber auch, dass sich einige Prüfungen des D. A. B. IV schneller mit anderen Reagenzien ausführen lassen, ohne an Schärfe zurückzutreten.

Da es mir nicht möglich ist, die Arbeit des Verfassers an dieser Stelle eingehend zu besprechen, so will ich nur einige der Versuche Wobbe's erwähnen; so fand er, dass sich fuchsinschweflige Säure als Reagens auf Aldehyd nicht anwenden lässt, da die Rotfärbung dieses Reagenses durch alle die Körper bedingt wird, welche die reduzierende Wirkung der schwefligen Säure aufheben, also besonders auch durch Wasserstoffsuperoxyd und Äthylperoxyd. Nach den Erfahrungen des Verfassers lassen sich Aldehyd und Vinylalkohol durch einfache Reaktionen nicht unterscheiden, als schärfstes Reagens auf beide Körper empfiehlt der Verfasser Nessler's Reagens; ausser Aldehyd und Vinylalkohol kann man auch Wasserstoffsuperoxyd und Weingeist mit diesem Reagens erkennen. Die Unterscheidung von Wasserstoffsuperoxyd und Äthylperoxyd kann man nach Wobbe mit Schöubein's Reagens (eine Mischung aus Ferri-

1) Zeitschrift d. allgem. österr. Apotheker-Vereins 58, 1165.

2) Pharmazeut. Zentral-Halle 44, 657. 